\title{
Highly Accurate CAD Tools for Cranial Implants
}

\author{
Kyoung-june $\operatorname{Min}^{1}$ and David Dean ${ }^{2}$ \\ ${ }^{1}$ Department of Electrical Engineering and Computer Science, \\ Case Western Reserve University, Cleveland, Ohio 44106 USA \\ kjm11@po.cwru.edu \\ ${ }^{2}$ Department of Neurological Surgery, University Hospitals of Cleveland, and \\ Case Western Reserve University, Cleveland, Ohio 44106 USA \\ dxd3 5@po. cwru . edu
}

\begin{abstract}
Prefabricated patient-specific cranial implants are especially useful for large cranial defects. We present CAD tools for large cranial implants that include validation of fit prior to rapid prototype fabrication. Our CAD process first determines whether the prototype implant surface intersects adjacent softtissue structures via collision detection. If so, we measure the volume of both the intersected dura and unoccupied area under the implant. These data are used to determine whether the prototype implant requires modification. If modification is necessary, we deform the prototype implant surface via a thin plate spline warp. Second, we interactively create and verify the implant taper surface that connects the external and internal implant surfaces. The taper surface seats the implant onto the patient's cranial defect site. The taper and patient's skull surfaces may contact but must not intersect one another. Results from tests on five patient data sets show our CAD and validation methods result in implant designs which are an improvement over, and not feasible by, current manual implant preparation methods.
\end{abstract}

\section{Introduction}

Cranial defects are usually repaired intra-operatively with malleable materials when the patient's own bone tissue is of insufficient quantity or quality. However, if the defect is sufficiently large $\left(>100 \mathrm{~cm}^{2}\right)$, pre-operative fabrication of a patient-specific implant may be considered. These implants should not only restore the patient's natural cranial aesthetic shape, but must also accurately fit the patient to prevent subsequent movement. In order to fabricate accurately fitting cranial implants, RP (Rapid Prototype) skull models based on 3D CT data have been used to manually fabricate implants for about a decade. Although clinical reports [1] indicate that these implants show satisfactory results, considerable cost is incurred, and time spent, producing full skull models and carrying out manual fabrication procedures.

As a fully digital alternative, a combination of computer aided design (CAD) and computer aided manufacture (CAM) technologies utilizing the patient's 3D CT scan have been reported $[2,3,4]$. These CAD/CAM methodologies significantly reduce

R.E. Ellis and T.M. Peters (Eds.): MICCAI 2003, LNCS 2878, pp. 99-107, 2003.

() Springer-Verlag Berlin Heidelberg 2003 
the time and cost of producing reasonably well-fitting thin plate or mesh, titanium cranial implants. However, partitioning of the implant surface is required when the cranial defect is sufficiently large, a procedure that is likely to result in surface curvature discontinuities (i.e., seams or gaps). Of concern is potential surface curvature discontinuity across the patient-to-implant boundary. To overcome these limitations, we have previously presented a top-down cranial implant CAD approach based on a two pass thin plate spine (TPS) warp [5] of deformable skull templates [6, 7]. The process includes the detection of a space curve, representing the cranial defect margin, surrounding a smooth implant surface, which has been directly partitioned from a skull template. A significant gap in published work on cranial implant CAD is the lack of consideration of the soft-tissue structures adjacent to the implant. It is imperative that the implant does not intersect nor compress the brain and that it sits safely below the scalp. Another methodological gap in the published work on cranial implant CAD is the lack of verification of implant-bone defect site contact (i.e., implant seating). Our clinical experience suggests that one must insure that the implant contacts, but does not intersect, the adjacent skull defect site.

In this paper, we present CAD tools that accurately assess large cranial implant fit prior to RP production. The initial cranial implant prototype is a surface without thickness that results from our previous work $[6,7]$. This surface corresponds to the external surface of the final implant design. Adding an internal surface parallel to this external surface, and then merging these two surfaces, introduces an additional surface. We refer to this connecting surface as the taper. The taper is where the implant will contact the patient's skull. Our CAD tools not only determine the quality of the prototype implant's fit, but also facilitate modification, if an intersection with the brain or skull is detected after the implant is designed. We detect and remove these intersections via a test we refer to as implant surface verification.

Solid or meshwork titanium cranial implant parts may be only $1.5 \mathrm{~mm}$ thick [4], and are often designed to partially overlap the defect site, possibly providing screw fixation holes. However, polymethylmehacrylate cranial implants usually have a thickness greater than $4 \mathrm{~mm}$, resulting in a taper. This creates the need for assessment of the taper-skull contact validation, what we refer to as implant taper verification. Although others have previously attempted to verify CAD/CAM fabricated titanium cranial implant seating using both skull images and models produced by RP [8], we are not aware of any other work that validates contact as well as non-intersection of the implant with the adjacent soft tissue or skull prior to fabrication. We use collision detection to facilitate the verification of non-intersection between the prototype implant design and a polygonal mesh surface derived from the patient's 3D CT image.

\section{Implant Surface Verification and Modification}

The primary objective of implant surface verification is to ensure that the implant surface does not intersect adjacent structures. To begin this process, we represent the prototype implant surface, $\mathbf{S}_{\mathrm{P}}$, and an additional surface representing the dura mater surface, $\mathbf{S}_{\mathrm{D}}$, obtained from $3 \mathrm{D} \mathrm{CT}$ as polygonal mesh object. The dura mater is a 
flexible fluid-filled layer wrapping the brain. Its shape may change following creation of the cranial defect due to brain swelling. A polygonal mesh surface representation of the dura mater is obtained by manual segmentation, followed by smoothing with a method we have previously presented [7].

\subsection{Implant Surface Verification Using Collision Detection}

We selected the RAPID algorithm, which uses an $O B B$ (Oriented Bounding Box) tree [9], as our collision detection algorithm for implant surface verification. RAPID collision detection is performed between a pair of polygonal soup objects, which are a collection of polygons without topological information. In our case, the RAPID algorithm detects intersections between the dura mater surface, $\mathbf{S}_{\mathrm{D}}$, and the prototype implant surface, $\mathbf{S}_{\mathrm{P}}$. If few or no collisions occur between $\mathbf{S}_{\mathrm{D}}$ and $\mathbf{S}_{\mathrm{P}}$ then, it is assumed that the implant prototype will safely occupy the sleeve between the dura mater and the scalp. However, these collisions are significant when more of the dura mater has been intersected than can fit under the implant. In this case, we will modify the prototype implant design so as to elevate the surface, thereby reducing the intersected volume to a level that can be accommodated underneath the cranial implant.

Before deciding to modify the prototype implant surface we analyze both the intersected dura mater volume and the intra-cranial volume available (i.e., unoccupied) between the implant and the brain. First, we place surfaces $\mathbf{S}_{\mathrm{D}}, \mathbf{S}_{\mathrm{P}}$, and the defect margin, $\mathbf{D}$, in the same reference frame. Then we calculate the normal vector, $\mathbf{n}$, of the best-fitting plane for $\mathbf{D}$. The points in $\mathbf{D}$ are sequentially ordered to form a closed contour. Next, we re-orient all objects so that $\mathbf{n}$ is aligned parallel to the Z-axis, and then define a plane, $\mathbf{P}_{\mathrm{G}}$, with normal vector $\mathbf{n}$, composed of $n \times m$ regularly spaced points. We cast a ray from each point on $\mathbf{P}_{\mathrm{G}}$ along direction $\mathbf{n}$ to build a $n \times m$ volume matrix $\mathbf{V}$ (Fig. 1). Finally, we store the positions of the ray-to-surface collisions detected on $\mathbf{S}_{\mathrm{D}}$ and $\mathbf{S}_{\mathrm{P}}$.

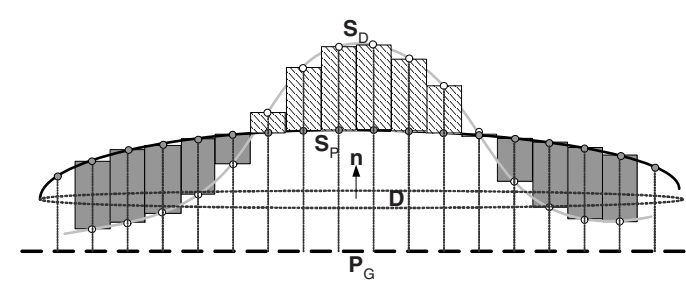

Fig. 1. Volume estimation of penetrated dura mater and space internal to the implant based on ray-to-surface collisions. Gray area is volume internal to the implant and hatched area is intersected dura mater volume. See text for full explanation of labels.

Once the homologous points in the two surfaces, $\mathbf{S}_{\mathrm{P}}$ and $\mathbf{S}_{\mathrm{D}}$, are mapped, the volume internal to the implant and the volume of the intersected dura mater are calculated via discrete definite integration. We fit 3D hexahedra between the corresponding points of $\mathbf{S}_{\mathrm{D}}$ and $\mathbf{S}_{\mathrm{P}}$. Figure 1 shows a cross-sectional view of these fitted hexahe- 
dra, where the cross section of each hexahedron is depicted as a rectangle. An interval along the ray that corresponds to space internal to the implant will collide first with $\mathbf{S}_{\mathrm{D}}$ and then $\mathbf{S}_{\mathrm{P}}$. In contrast, an interval that corresponds to an intersected portion of the dura mater will collide with $\mathbf{S}_{\mathrm{P}}$ first and then $\mathbf{S}_{\mathrm{D}}$. The $n \times m$ matrix $\mathbf{V}$ has elements $\mathbf{V}_{i j}$ at the $i^{\text {th }}$ row and the $j^{\text {th }}$ column derived from the ray casting procedure according to two rules: First, if less than one collision occurs at the $i^{\text {th }}$ row and the $j^{\text {th }}$ column point, then we set $\mathbf{V}_{i j}=0$. Second, if two collisions occur at the $i^{\text {th }}$ row and the $j^{\text {th }}$ column point of ray cast grid plane, $\mathbf{P}_{\mathrm{G}}$, then we set $\mathbf{V}_{i j}=z_{s p}-\cdot z_{s d}$, where $z_{s p}$ is the $\mathrm{z}$ component of the collision position on $\mathbf{S}_{\mathrm{P}}$ and $z_{s d}$ is that on $\mathbf{S}_{\mathrm{D}}$. A constant $(\geq 1)$ is a scaling factor. The equation used to determine the need the revision of a prototype implant surface is defined as:

$$
d=\sum_{i, j} \mathbf{V}_{i j}
$$

We refer to $d$ as the modification coefficient, and modify the prototype implant surface only if $d$ has a positive value. The scaling factor increase the weight of the penetrated dura mater volume, thereby penalizing implant space internal to the implant. This is necessary since the dura matter and underlying brain are not truly elastic. Note that when $=1$, the approximated difference of the two original volumes can be calculated as $\mathrm{V}=d w h$, where $w$ and $h$ are the width and height of each hexahedral grid element, respectively. Thus, the sign of $d$ indicates whether the larger volume occurs internal to the implant or externally in the intersected dura mater.

\subsection{Implant Surface Modification Using TPS Warp}

If the value of $d$ turns out to be negative, we deform $\mathbf{S}_{\mathrm{P}}$ via a TPS warp [5]. The homologous surface point pairs that were identified during the volume calculation process in $\mathbf{S}_{\mathrm{D}}$ and $\mathbf{S}_{\mathrm{P}}$ are used for this warp. We set points on $\mathbf{S}_{\mathrm{D}}$ as the target warp points for the corresponding source warp points on $\mathbf{S}_{\mathrm{P}}$. Along with these source and target configurations, we set invariant points, that we refer to as anchor warp points, around the implant rim and the non-intersected area. These anchor warp points impose a constraint preventing prototype implant shape change that would compromise implant fixation or proper surface convexity.

\section{Implant Taper Creation and Verification}

After the final external implant surface is obtained, we define the internal implant surface and the implant taper. The taper will contact the cranial defect site and also closes the external and internal implant polygonal mesh surface, resulting in a CAD object with a closed surface that is acceptable for RP production. 


\subsection{Determining the Location and Size of the Internal Implant Surface}

We define the internal implant surface, $\mathbf{S}_{2}$, by duplicating $\mathbf{S}_{1}$, and applying a geometric transformation of $\mathbf{p}_{\mathrm{S} 2}=\mathbf{T}_{\mathrm{T}} \mathbf{T}_{\mathrm{S}} \mathbf{p}_{\mathrm{S} 1}$, where $\mathbf{p}_{\mathrm{S} 2}$ is the point set composing $\mathbf{S}_{2}$, and $\mathbf{p}_{\mathrm{S} 1}$ is the point set of $\mathbf{S}_{1}$. A scaling matrix $\mathbf{T}_{\mathrm{S}}$ first shrinks the internal surface and a translation matrix $\mathbf{T}_{\mathrm{T}}$ shifts the internal surface so that it lies below the external surface. The direction of the translation is parallel to the normal vector of the best-fitting plane of D. Note that $\mathbf{S}_{1}$ and $\mathbf{S}_{2}$ have identical topological structures, thereby allowing creation of a triangle stripe linking the homologous points of the two aligned rims (Fig. 2). To analyze the nature of the contact (i.e., seating) between the taper and the cranial defect site on the patient's skull, we divide the taper surface into four polygonal stripe layers [Fig. 2(a)]. Then, each stripe layer is assigned to a separate group of triangles for RAPID collision detection with the cranial defect site.

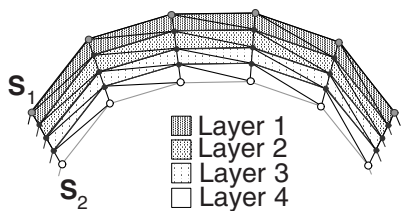

(a)

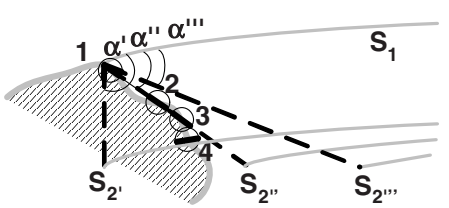

(b)

Fig. 2. Taper angle determination based on collision pattern. (a) Subdivision of the taper into four layers. (b) From a single external implant surface $\mathbf{S}_{1}$, the internal implant surfaces $\mathbf{S}_{2 \cdot}, \mathbf{S}_{2 . .}$, and $\mathbf{S}_{2} \ldots$ can be defined depending on the scaling factor chosen for $\mathbf{S}_{2}$.

\subsection{Implant Taper Non-intersection Verification and Modification}

The taper must contact the cranial defect site, but should not penetrate it. We employ the RAPID collision detection algorithm to numerically and visually determine the degree of contact between the taper surface and the patient's cranial defect site.

Figure 2(b) depicts intersecting regions between the potential taper and the cranial defect site as detected by the RAPID algorithm. The shaded region represents the bony tissue structure of the cranial defect site, where $\mathbf{S}_{2 .}, \mathbf{S}_{2 . .}$, and $\mathbf{S}_{2 \ldots}$ are possible internal implant surfaces generated from decreasing scale factors. We denote angle , found between $\mathbf{S}_{1}$ and the taper, as the taper angle. Without losing contact at the external implant surface, we see that the possibility of penetration decreases proportional to . If the scaling factor is too large (i.e., angle •), the taper becomes too steep. This causes the taper (dashed line) to penetrate the patient's cranial defect site. In this case, collisions are detected in circled regions 1 and 4 , where region 4 is located on the internal implant surface $\mathbf{S}_{2}$. In contrast, if the scaling factor is too small (i.e., angle $\bullet \bullet)$, a shallow taper produces a surface contact only in circled region 1 . This may result in a loose fit. Finally, an optimal scaling factor (i.e., angle $\bullet$ ) results in collisions at circled regions 1,2 , and 3 . A pattern showing no collisions at $\mathbf{S}_{2}$, with decreasing frequency of collisions as the taper gets closer to the $\mathbf{S}_{2}$, is the desired situa- 
tion as seen on taper $\mathbf{S}_{1}-\mathbf{S}_{2 .}$ of Figure 2(b). Note that the taper angle is dependent on both the scaling and the translation factors. The percentage of area contacting the cranial defect in each layer is calculated by counting the number of collided triangles versus the total number of triangles composing the stripe.

\section{Results}

We implemented and tested methods presented above on a Silicon Graphics Inc. (Palo Alto, CA) Octane Workstation with a R10000 CPU and 256M bytes of RAM. The Open Inventor class libraries and IRIS Viewkit were used for interactive 3D rendering and user interface implementation. All algorithms were tested on five different patient skull 3D CT data sets presenting defects of various sizes and locations. Polygonal mesh skull images were produced via the Wrapper algorithm [10].

\subsection{Implant Surface Verification and Modification}

The prototype implant surface verification of patient 03004 is shown in Figure 3 . Note that the implant prototype surface and the dura mater surface are both segmented from the same patient's 3D CT volume-scan, requiring no additional 3D image registration. Collision detection between the prototype implant surface (light area) and the dura mater surface (dark) presents significant surface intersections [Fig. 3(a)]. The light contour highlights intersecting triangles in the prototype implant surface, while the dark indicates those in the dura mater surface. Based on the indication of a large intersection, we performed a volume calculation of the intersected dura mater [Fig. 3(b)] by summing the light hexahedra. The dark hexahedra sum the open space internal to the implant. The resulting modification coefficient was $d=-207$, where we preset $=3$. Because $d$ was negative, we used 397 points, including anchor warp points and defect margin contour points, to drive a compensatory TPS warp of the prototype implant surface. We compared the contact of the implant prototype for patient 03004 before [Fig.3 (c)] and after [Fig.3 (d)] the implant surface modification.

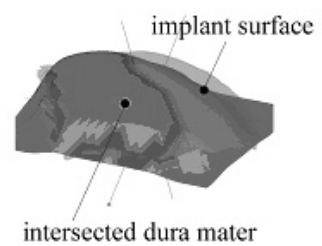

(a)

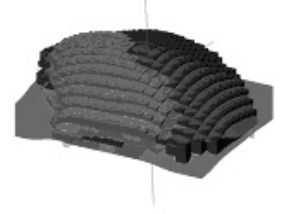

(b)

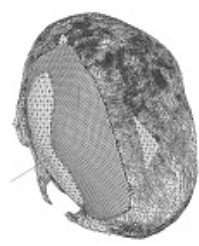

(c)

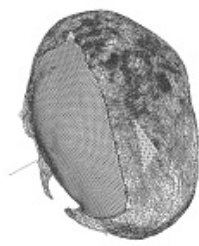

(d)

Fig. 3. Implant surface verification and modification for patient 03004. (a) Collision detection between the prototype implant and the dura mater surface. (b) Volume analysis. (c) Collision detection between implant, dura mater, and patient's skull surface before modification. Light contour indicates boundary of intersected dura mater. Dark contour indicates collisions in prototype implant rim. (d) Collision detection of the same objects as in (c) after modification. 


\subsection{Implant Taper Verification for All Five Patient Data Sets}

Table 1 presents the results for implant taper verification for five patient data sets, including the percentage of collisions at each stripe layer of the taper, and the scaling and translation factors. The first taper polygonal stripe layer, adjacent to the external implant surface, presents approximately 40 to $50 \%$ of the contact area. This decreases to less than $3 \%$ when appropriate scaling and translation parameters are chosen.

Table 1. Implant taper to skull contact verification. From column two through four, percent of surface contact in each stripe layer of the taper is shown. The last two columns show translation and scale factors that define the internal implant surface and taper angle.

\begin{tabular}{cccccc}
\hline Patient ID & 03003 & 03004 & 03005 & 03008 & 03010 \\
\hline $1^{\text {st }}$ Layer Col. (\%) & 44 & 55 & 39 & 49 & 50 \\
$2^{\text {nd }}$ Layer Col. (\%) & 33 & 30 & 41 & 36 & 22 \\
$3^{\text {rd }}$ Layer Col. (\%) & 36 & 6 & 33 & 6 & 4 \\
$4^{\text {th }}$ Layer Col. (\%) & 3 & 1 & 1 & 2 & 1 \\
Translation Factor & 5.0 & 7.8 & 5.7 & 19.4 & 27.9 \\
Scaling Factor (\%) & 79 & 86 & 81 & 79 & 79 \\
\hline
\end{tabular}

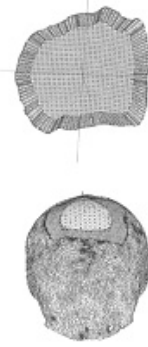

(a)
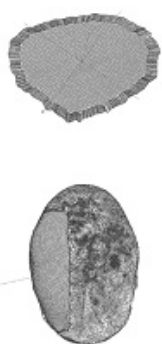

(b)
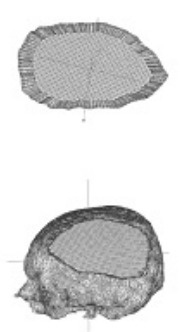

(c)
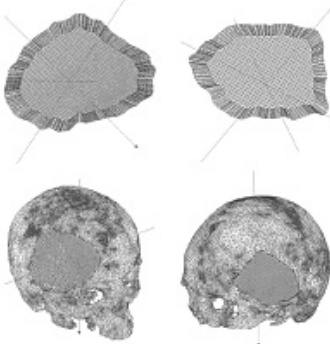

(d)

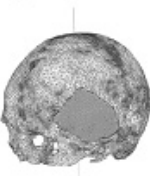

(e)

Fig. 4. Final cranial implant fit. (a) Patient 03003. (b) Patient 03004. (c) Patient 03005. (d) Patient 03008. (e) Patient 03010. Light triangles show contacted polygons on the implant surface, and dark triangles show contacted polygons on the patient's dura mater or skull surface.

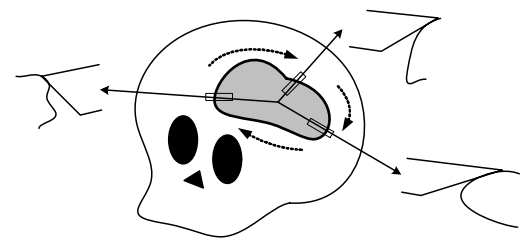

Fig. 5. Adaptive tapering could define a different taper radially around the defect margin. 
Figure 4 shows the final cranial implant specifications that indicate contact (light triangles) regions with the cranial defect site in the top row, and the contacted cranial and dura mater surfaces (dark triangles) in the bottom row. Note that taper contacts decrease from external to internal, showing no collisions at the internal implant surface and, overall, no intersections between the taper and the cranial defect site. While, both the translation and scaling factors can be interactively altered by the operator, the translation factor directly controls the resulting implant thickness which is specified by the surgeon.

\section{Discussion}

The results suggest that our cranial implant CAD approach will result in a useful implant model if it is rendered in a RP device. A few issues arose during our research. While our visually interactive taper determination approach is practical, the implant designer must make a random initial guess to decide the scale factor. Instead, this process could be modeled as an optimization of the scaling factor to obtain the maximum acceptable taper angle. Moreover, our taper determination method produces a uniform taper angle, not allowing region-specific contact optimization. Alternatively, an adaptive taper determination could analyze implant-patient contact radially around the defect margin (Fig. 5). It is important that the taper rest on and interlock with the defect. Finally, future implant CAD software could consider biomechanical performance as well as the behavior of biocompatible materials among the design parameters.

\section{References}

1. Voigt, M., Schaefer, D. J., Andree, C.: Three-dimensional reconstruction of a defect of the frontozygomatic area by custom made Proplast II implant, Eur. J. Plast. Surg. 23 (2000) 391-394

2. Wehmöller, M., Eufnger, H., Kruse, D., Ma berg, W.: CAD by processing of computed tomography data and CAM of individually designed prostheses, Int. J. Oral Max. Surg. 24 (1995) 90-97

3. Carr, J. C., Fright, W. R., Beatson, R. K.: Surface interpolation with radial basis functions for medical imaging, IEEE T. Med. Imaging 16 (1997) 96-107

4. Eufinger, H., Saylor, B.: Computer-Assisted Prefabrication of Individual Craniofacial Implants, AORN J. 74 (2001) 648-654

5. Bookstein, F. L.: Principal Warps: Thin-Plate Splines and the Decomposition of Deformations, IEEE T. Pattern Anal. 11 (1989) 567-585

6. Dean, D., Min, K. J., Bond, A.: Computer Aided Design of Pre-Fabricated Cranial Plates, J. Craniofac. Surg. In Press (2003)

7. Min, K. J.: Computer Aided Design of Cranial Implants Using Deformable Templates, Ph.D. Thesis, Case Western Reserve University, Cleveland Ohio (2003) 
8. Joffe, J. M., Nicoll, S. R., Richards, R., Linney, A. D., Harris, M.: Validation of computer assisted manufacture of titanium plates for cranioplasty, Int. J. Oral. Max. Surg. 28 (1999) 309-313

9. Gottschalk, S., Lin, M. C., Manocha, D.: OBB-Tree: A hierarchical structure for rapid interference detection, ACM SIGGRAPH 96 Conference Proceedings, Annual Conference Series, ACM SIGGRAPH, Addison Wesley, New Orleans Louisiana (1996) 171180

10. Gueziec, A., Hummel, R.: Exploiting triangulated surface extraction using tetrahedral decomposition, IEEE T. Vis. Comput. Graphics 1 (1995) 328-342 